\title{
The differential requirement of mushroom body $\alpha / \beta$ subdivisions in long-term memory retrieval in Drosophila
}

\author{
Cheng Huang ${ }^{1,3}$, Pengzhi Wang ${ }^{1,4}$, Zhiyong Xie ${ }^{1}$, Lianzhang Wang ${ }^{1}, Y_{i}$ Zhong $^{1,2, 凶}$ \\ ${ }^{1}$ School of Life Sciences, Tsinghua University, Beijing 100084, China \\ ${ }^{2}$ Cold Spring Harbor Laboratory, Cold Spring Harbor, NY 11724, USA \\ ${ }^{3}$ Present address: Department of Biological Sciences, Stanford University, Stanford, CA 94305, USA \\ ${ }^{4}$ Present address: Department of Bioengineering, University of California, San Diego, La Jolla, CA 92093, USA \\ $\triangle$ Correspondence: zhongyi@cshl.org
}

Received May 1, 2013 Accepted May 14, 2013

\begin{abstract}
The mushroom body (MB), a bilateral brain structure possessing about 2000-2500 neurons per hemisphere, plays a central role in olfactory learning and memory in Drosophila melanogaster. Extensive studies have demonstrated that three major types of MB neurons $\left(\alpha / \beta, \alpha^{\prime} / \beta^{\prime}\right.$ and $\left.\gamma\right)$ exhibit distinct functions in memory processing, including the critical role of approximately $1000 \mathrm{MB} \alpha / \beta$ neurons in retrieving long-term memory. Inspired by recent findings that $M B \alpha / \beta$ neurons can be further divided into three subdivisions (surface, posterior and core) and wherein the $\alpha / \beta$ core neurons play an permissive role in long-term memory consolidation, we examined the functional differences of all the three morphological subdivisions of MB $\alpha / \beta$ by temporally precise manipulation of their synaptic outputs during long-term memory retrieval. We found the normal neurotransmission from a combination of $M B \alpha / \beta$ surface and posterior neurons is necessary for retrieving both aversive and appetitive long-term memory, whereas output from MB $\alpha / \beta$ posterior or core subdivision alone is dispensable. These results imply a specific requirement of about $500 \mathrm{MB} \alpha / \beta$ neurons in supporting long-term memory retrieval and a further functional partitioning for memory processing within the $M B \alpha / \beta$ region.
\end{abstract}

KEYWORDS memory retrieval, neural circuits, aversive olfactory conditioning, appetitive olfactory conditioning, mushroom body

\section{INTRODUCTION}

Memory is of a complex and dynamic nature, requiring the co- ordination of different neuron ensembles to support its distinct stages (i.e. formation, consolidation and retrieval), so dissecting the neural circuits underlying is crucial for mechanistically understanding the memory processing and the pathological basis of neurological diseases associated with memory deficits (Silva et al., 2009; van Strien et al., 2009; Small et al., 2011; Tye and Deisseroth, 2012). In Drosophila, with the power of sophisticated genetic toolbox and behavioral paradigm, extensive studies suggest a dynamic requirement for the three major subtypes of mushroom body (MB) neurons in olfactory conditioning: $\mathrm{y}$ neurons server as a gateway to support memory formation by dopaminergic signal-mediated CS-US association (Blum et al., 2009; Qin et al., 2012), $\alpha^{\prime} / \beta^{\prime}$ neurons were transiently required to stabilize memory after training (Krashes et al., 2007) and to retrieve the immediate memory (Wang et al., 2008), and the outputs of $\alpha / \beta$ neurons are necessary for retrieving both aversive and appetitive long-term memory (LTM) (Isabel et al., 2004; Krashes et al., 2008; Trannoy et al., 2011).

Through detailed characterization of enhancer trap lines, $\alpha / \beta, \alpha^{\prime} / \beta^{\prime}$ and $y$ can be further divided into more specific subdivisions. For instance, the $\alpha / \beta$ neurons are consist of surface, posterior and core subdivisions (Aso et al., 2009; Tanaka et al., 2008). In addition, recent literatures also reported that different expression patterns of genes within $\alpha / \beta$ subdivisions, such as DmGluRA (the only Drosophila mGluR) and Ago-3 (an Argonaute protein) are selectively expressed in $\alpha / \beta$ core neurons (Sinakevitch et al., 2010; Perrat et al., 2013), whereas the NMDA receptor subunit dNR1 and short NPF are exclusively expressed in non-core $\alpha / \beta$ neurons (Sinakevitch et al., 2010; Johard et al., 2008). Given the well-known postulation "form follows function", the observations above may imply the existence of functional differences among subdivisions of $M B \alpha / \beta$ 
$P\{G A L 4\}>$ UAS-mCD8:: GFP;MB247-DsRed
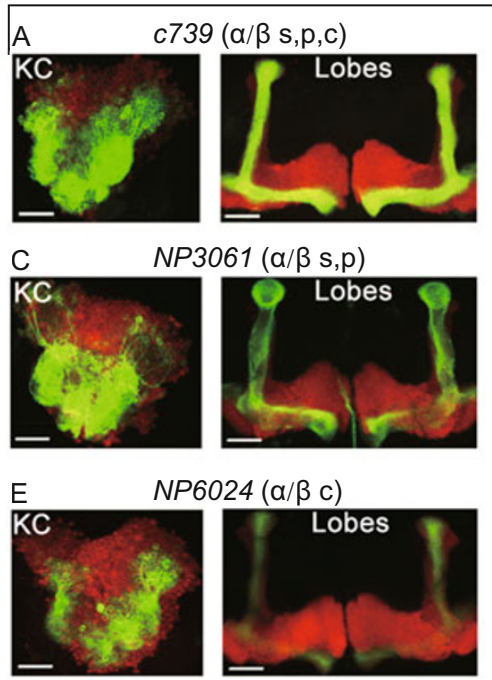

NP6024 ( $\alpha / \beta$ c)

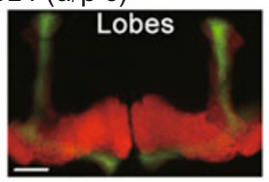

Figure 1. Expression patterns in $M B \alpha / \beta$ subdivisions of six GAL4 enhancer trap lines. Indicated GAL4 lines were crossed to flies carrying both UAS$m C D 8:: G F P$ and MB247-DsRed transgenes. Confocal imaging of whole-mount adult central brain reveals the MB structure (displayed by MB247-DsRed, red signal) and GAL4 expression (indicated by GFP, green signal) in both Kenyon Cell region (KC) (left panel) and $\mathrm{MB}$ Lobes region (right panel). (A) $c 739$ had a strong GFP expression in the whole $M B \alpha / \beta$ region, including all three subdivisions (s: surface, p: posterior and c: core neurons). Scale bar is $20 \mu \mathrm{m}$. (B and C) Both NP5286 and NP3061 labeled MB $\alpha / \beta$ surface and posterior neurons, but not core subdivision. Scale bar is $20 \mu \mathrm{m}$. (D) NP3208 exhibited a specific expression in MB $\alpha / \beta$ posterior subdivision. Scale bar is $20 \mu \mathrm{m}$. (E) NP6024 labeled both the inner and outer neurons of MB $\alpha / \beta$ core subdivision. Scale bar is $20 \mu \mathrm{m}$. (F) NP7175 specifically labeled the inner MB $\alpha / \beta$ core subdivision. Scale bar is $20 \mu \mathrm{m}$.

neurons

In the current work, we tested necessity of the synaptic output from different MB $\alpha / \beta$ subdivisions during LTM retrieval. Here we show that a subgroup of about $500 \mathrm{MB} \alpha / \beta$ neurons, instead of the whole $\mathrm{MB} \alpha / \beta$ region ( 1000 neurons) suggested before (Isabel et al., 2004; Krashes et al., 2008; Trannoy et al., 2011), is critical for successfully retrieving both aversive and appetitive LTM. Combined with the previous finding that $\alpha / \beta$ core neurons play a distinct role in gating LTM consolidation (Huang et al., 2012), we propose that a further functional partitioning within $M B \alpha / \beta$ region in LTM processing.

\section{RESULTS}

\section{A panel of GAL4 enhancer trap lines marks MB $\alpha / \beta$ subdivisions}

According to gene expression patterns and morphological structures, about 2000-2500 intrinsic MB Kenyon cells (Aso et al., 2009; Heisenberg, 2003) can be classified into three major subtypes- $\alpha / \beta, \alpha^{\prime} / \beta^{\prime}$ and $y$ neurons (Crittenden et al., 1998; Lee et al., 1999) and each subtype can be further divided into smaller subdivisions such as the posterior, surface, and core neurons in $M B \alpha / \beta$ region (Tanaka et al., 2008; Aso et al., $2009)$. Even though a wealth of evidence suggests that $M B$ $\alpha / \beta$ neurons are critical in retrieving both aversive and appetitive LTM (Isabel et al., 2004; Krashes et al., 2008; Trannoy et al., 2011), our recent finding revealed the $\alpha / \beta$ core subdivision permits LTM consolidation, which is functionally distinct from the other two $\alpha / \beta$ subdivisions (Huang et al., 2012). In order to investigate the functional heterogeneity of $M B \alpha / \beta$ subdivisions, we chose six established GAL4 lines that are reported with the expression patterns covered all three $\alpha / \beta$ subdivisions. A close examination of confocal images from this panel of GAL4 lines with co-expressing mCD8::GFP and MB247-DsRed (outlines the MB structure) indicated c739 strongly expressed

in the whole $M B \alpha / \beta$ region including all the three subdivisions (Fig. 1A), while NP5286 (Fig. 1B) and NP3061 (Fig. 1C) both have a strong expression in $\alpha / \beta$ surface neurons and labeled posterior subdivision more weakly as reported (Tanaka et al., 2008). Also, posterior neurons can be marked by NP3208 specifically (Fig. 1D), while core neurons are labeled by NP6024 (Fig. 1E) or NP7175 (Fig. 1F). It is worth noting that no specific $\alpha / \beta$ surface driver is available to our knowledge, but the combinational expression of this panel of GAL4 lines still provides us a great opportunity to probe the different functions of $M B \alpha / \beta$ subdivisions.

A combination of $M B \alpha / \beta$ surface and posterior neurons is necessary for retrieving aversive LTM

In order to dissect the requirement of different $M B \alpha / \beta$ subdivisions in aversive LTM retrieval, we measured the memory performance at $24 \mathrm{~h}$ after spaced aversive training (reinforced by electric shock punishment) (Tully et al., 1985, 1994) and used $U A S-s h i^{t s 1}$, the temperature-sensitive shibire mutant transgene, to manipulate synaptic output reversibly and temporal precisely (Kitamoto, 2001). At the permissive temperature (below $23^{\circ} \mathrm{C}$ ), the mutated Dynamin encoded by $s h i^{\text {ts } 1}$ gene allows normal neurotransmitter release, whereas, at the restrictive temperatures (above $30^{\circ} \mathrm{C}$ ), it blocks synaptic transmission (Kitamoto, 2001).

All flies were subjected to two training procedures (Fig. 2A). For non-HS group, flies were trained and tested at the permissive temperature $\left(23^{\circ} \mathrm{C}\right)$ and it turned out that they could perform normal LTM at this circumstance (Fig. 2B-F). For HS group, flies were tested at the restrictive temperatures $\left(30^{\circ} \mathrm{C}\right)$. As shown in Fig. 2B, blockage of the synaptic output from c739 labeled neurons during testing disrupted the normal LTM performance, and the involvement of non-MB neurons in c739 could be ruled out by introducing $M B-G a / 80$ to eliminate the 
Aversive olfactory conditioning

Non-HS group

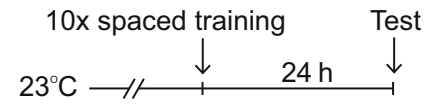

HS group:

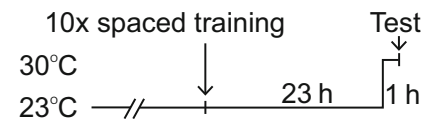

C $\square$ UAS-shits1/+

$\alpha / \beta$ surface and posterior (NP5286)

$\square N P 5286 /+$; UAS-shits1/+ $\quad \square N P 5286 /+$

$\square N P 5286 / M B-G A L 80 ;$ UAS-shits1/+ $\square$ NP5286/MB-GAL80

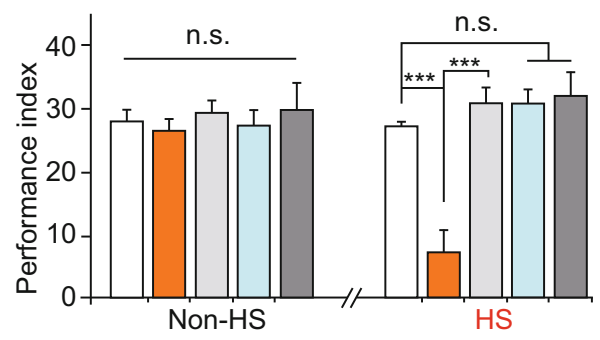

B
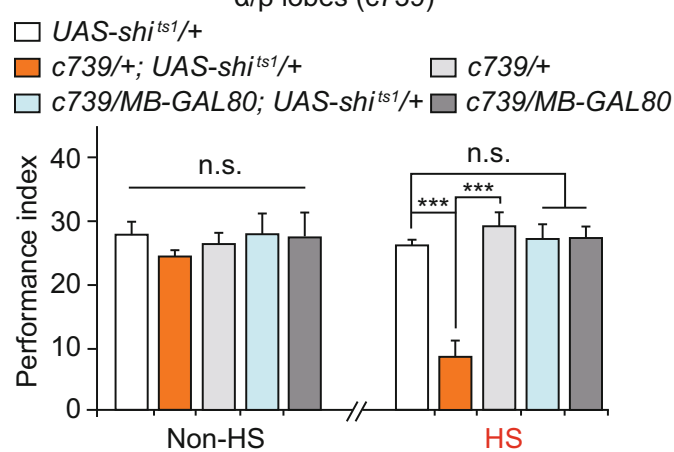

D $\square U A S-s h i t s 1 /+$

$\square N P 3061 /$ UAS-shits1 $\quad \square N P 3061 /+$

$\square M B-G A L 80 /+;$ NP3061/UAS-shits1 $\square$ MB-GAL80/+; NP3061/+

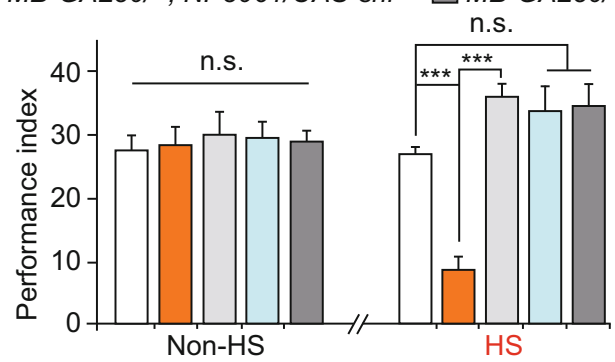

E

$\alpha / \beta$ posterior (NP3208) and core (NP6024, NP7175)

$\square+/+, ;$ UAS-shit ${ }^{t s 1} /+$

$\square$ NP3208/+;; UAS-shits1/+ $\square$ NP6024/+;; UAS-shit $1 /++\square N P 7175 /+; ;$ UAS-shits1/+

$\square N P 3208 /+$

$N P 6024 /+$

NP7175/+

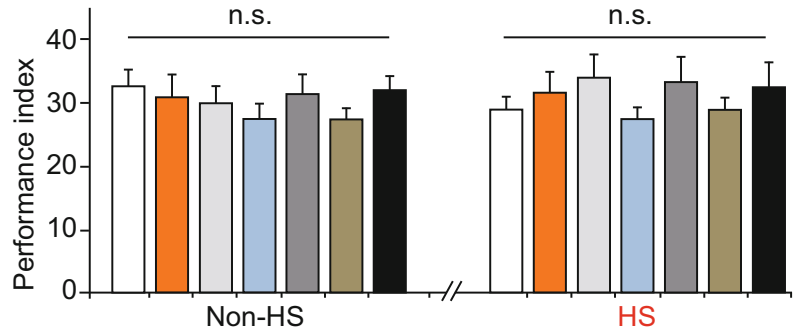

Figure 2. The synaptic output from a combination of $M B \alpha / \beta$ surface and posterior subdivisions is required for retrieving aversive LTM. (A) Temperature-shift protocols for non-HS and HS groups. For non-HS group, flies were trained by ten-session spaced aversive training and tested in $23^{\circ} \mathrm{C}$. For HS group, flies were trained and rested for $23 \mathrm{~h}$ at $23^{\circ} \mathrm{C}$, then transferred to $30^{\circ} \mathrm{C}$ for $1 \mathrm{~h}$ resting followed by testing.(B) $c 739$ labeled MB $\alpha / \beta$ region is necessary for aversive LTM retrieval. In HS group, blocking synaptic output from $c 739$ labeled neurons disrupted aversive LTM retrieval $(P<0.001, n=8-10$, ANOVA), while blocking non-MB $c 739$ neurons (by co-expressing MB-Gal80 and UAS-shits ${ }^{t s}$ ) had no effect $(P>0.99, n=8-10$, ANOVA). In non-HS group, all genotypes showed normal LTM performance $(P>0.71$, $n=8-10$, ANOVA and Kruskal-Walis ANOVA). (C) NP5286 labeled neurons (MB $\alpha / \beta$ surface and posterior) are required for aversive LTM retrieval. In HS group, blocking synaptic output from NP5286 labeled neurons disrupted aversive LTM retrieval $(P<0.001, n=8-10$, ANOVA), while blocking non-MB NP5286 neurons (by co-expressing MB-Gal80 and UAS-shit ${ }^{\text {ts }}$ ) had no effect $(P>0.49, n=8-10$, ANOVA). In non-HS group, all genotypes showed normal LTM performance $(P>0.93, n=8-10$, ANOVA and Kruskal-Walis ANOVA). (D) NP3061 labeled neurons (MB $\alpha / \beta$ surface and posterior) are required for aversive LTM retrieval. In HS group, blocking synaptic output from NP3061 labeled neurons disrupted aversive LTM retrieval $(P<0.001, n=8-10$, ANOVA), while blocking non-MB NP3061 neurons (by co-expressing MB-Gal80 and UAS-shit ${ }^{t s 1}$ ) had no effect $(P>0.23, n=8-10$, ANOVA). In non-HS group, all genotypes showed normal LTM performance $(P>0.99, n=8$, ANOVA). (E) Output from MB $\alpha / \beta$ posterior or core neurons is dispensable for aversive LTM retrieval. In HS group, blocking synaptic output from MB $\alpha / \beta$ posterior subdivision (labeled by NP3208) and core subdivision (labeled by NP6024 or NP7175) alone had no effect on LTM retrieval $(P>0.40, n=8-10$, ANOVA). In non-HS group, all genotypes showed normal LTM performance $(P>0.29, n=8-10$, ANOVA). For the $\mathrm{X}$ chromosome-located NP3208, NP6024 and NP7175, only female results are shown. Average data are presented as means \pm SEM, ${ }^{\star \star \star} P<$ 0.001 , n.s. for non-significance. 
$s h i^{t s 1}$ expression in Kenyon cells (Krashes et al., 2007). This observation confirmed the conclusion that $M B \alpha / \beta$ region is critical for retrieving aversive LTM as reported before (Isabel et al., 2004). Next, we demonstrated that blocking neural outputs from either NP5286 or NP3061 labeled neurons could impair aversive LTM retrieval, while LTM was normal by co-expression of $M B-G a / 80$ (Fig. 2C and 2D), implying a necessity of the combination of $\mathrm{MB} \alpha / \beta$ surface and posterior neurons in retrieving LTM. Importantly, those LTM impairments observed in c739, NP5286 and NP3061 flies were not the result of any change in sensory responses that are essential for the memory task (Table 1). Surprisingly, disrupting neurotransmission of NP3208 labeled MB $\alpha / \beta$ posterior neurons alone has no effect on retrieving aversive LTM (Fig. 2E). Moreover, in consistence with our previous report (Huang et al., 2012), outputs from $\alpha / \beta$ core neurons are dispensable for the read-out of aversive LTM (Fig. 2E).

A combination of $M B \alpha / \beta$ surface and posterior neurons is also required for appetitive LTM retrieval

In the appetitive memory task, we measured memory performance at $24 \mathrm{~h}$ after single appetitive training (reinforced by sugar rewarding) (Krashes et al., 2008). Since appetitive memory is controlled by fly's motivational states and can only be formed or retrieved when flies are hungry (Krashes et al., 2008 and 2009), all flies were food-deprived for $36 \mathrm{~h}$ before and 24 $\mathrm{h}$ after training (Fig. 3A). Control experiments (non-HS group) showed that all genotypes tested exhibited a normal appetitive LTM performance (Fig. 3B-F). We then demonstrated that, in HS-group, disrupting synaptic output from c739 labeled MB $\alpha / \beta$ neurons impaired appetitive LTM retrieval (Fig. 3B), which is in consistent with previous reports (Isabel et al., 2004). Likewise, blocking of $\mathrm{MB} \alpha / \beta$ surface and posterior neurons, labeled by NP5286 and NP3061, during testing could induce LTM deficits, which can be reversed by the existence of $M B-G a / 80$ in those GAL4 lines (Fig. 3C and 3D). The task-relevant sensori-motor responses of $c 739, N P 5286$ and NP3061 flies were not signifi- cantly altered (Table 1). However, appetitive LTM was normal in flies when synaptic transmission was blocked in the NP3208 labeled $\alpha / \beta$ posterior neurons and NP6024 or NP7175 labeled $\alpha / \beta$ core region (Fig. 3E).

\section{Rutabaga adenylyl cyclase is sufficient in NP5286 and NP3061 labeled MB subdivisions for supporting appetitive LTM}

rutabage (rut) gene encoded Calcium/calmodulin-dependent adenylyl cyclase is necessary to both aversive and appetitive LTM formation (Blum et al, 2009; Trannoy et al, 2011). Interestingly, restoring rut signaling in $\mathrm{MB} \alpha / \beta$ neurons is sufficient to fully rescue appetitive LTM defect (Trannoy et al, 2011), suggesting a predominant role of those neurons in rut-dependent LTM formation. Because shibire blocking experiments indicating $M B \alpha / \beta$ subdivisions involve differently in LTM processing, we investigated if rut signaling in $M B \alpha / \beta$ subdivision could be sufficient to support appetitive LTM. We found appetitive LTM defect in rut ${ }^{2080}$ mutant could be fully rescued by expressing UAS-rut into NP5286 and NP3061 labeled MB subdivisions as well as restoring rut signaling in c739 labeled neurons (Fig. 4).

Taken together, our results showed that, for both aversive and appetitive LTM retrieval, the normal neurotransmission from a combination of $\mathrm{MB} \alpha / \beta$ surface and posterior neurons is necessary, whereas output from $\alpha / \beta$ posterior or core subdivisions alone is totally dispensable. Moreover, rut-dependent signaling in this combination of $M B \alpha / \beta$ neurons is sufficient to support LTM formation.

\section{DISCUSSION}

The current work revisited the role of $\alpha / \beta$ neurons in LTM retrieval and tested if any functional difference exists in the individual morphological subdivision of $M B \alpha / \beta$ neurons. To do so, we used a panel of established GAL4 enhancer trap lines that faithfully label the $\alpha / \beta$ subdivisions in a combinational manner and target neurons of interest with UAS-shit ${ }^{\text {ts } 1}$ to manipulate their synaptic transmission temporally. Since all of GAL4 lines

Table 1. Task-relevant sensori-motor abilities

\begin{tabular}{|c|c|c|c|c|c|c|c|}
\hline \multirow{2}{*}{ Genotype } & \multicolumn{2}{|c|}{ OCT $\left(1.5 \times 10^{-3}\right)$} & \multicolumn{2}{|c|}{$\mathrm{MCH}\left(1.0 \times 10^{-3}\right)$} & \multicolumn{2}{|c|}{ Sucrose acuity } & \multirow{2}{*}{$\begin{array}{c}\text { Shock reactivity } \\
23^{\circ} \mathrm{C}\end{array}$} \\
\hline & $23^{\circ} \mathrm{C}$ & $30^{\circ} \mathrm{C}$ & $23^{\circ} \mathrm{C}$ & $30^{\circ} \mathrm{C}$ & $23^{\circ} \mathrm{C}$ & $30^{\circ} \mathrm{C}$ & \\
\hline$U A S-s h t^{t s 1} /+$ & $47.7 \pm 5.8$ & $47.7 \pm 5.9$ & $44.0 \pm 5.1$ & $48.3 \pm 4.7$ & $34.8 \pm 4.5$ & $40.5 \pm 5.4$ & $70.4 \pm 3.7$ \\
\hline c739/+ & $52.7 \pm 3.1$ & $48.7 \pm 5.7$ & $45.2 \pm 3.7$ & $41.1 \pm 5.2$ & $33.4 \pm 3.8$ & $42.7 \pm 4.9$ & $73.4 \pm 3.8$ \\
\hline c739/+; UAS-shis1/+ & $47.1 \pm 3.2$ & $49.0 \pm 6.4$ & $40.5 \pm 3.7$ & $48.1 \pm 6.2$ & $39.3 \pm 4.5$ & $45.2 \pm 6.2$ & $68.7 \pm 3.4$ \\
\hline NP5286/+ & $55.2 \pm 3.6$ & $49.7 \pm 5.1$ & $44.9 \pm 4.8$ & $49.6 \pm 6.4$ & $35.2 \pm 4.7$ & $35.6 \pm 5.8$ & $70.4 \pm 5.6$ \\
\hline NP5286/+; UAS-shits1/+ & $52.4 \pm 4.7$ & $48.1 \pm 4.8$ & $39.2 \pm 4.0$ & $47.9 \pm 7.4$ & $39.8 \pm 5.3$ & $36.6 \pm 5.4$ & $68.9 \pm 3.0$ \\
\hline NP3061/+ & $47.8 \pm 2.0$ & $45.0 \pm 7.0$ & $39.2 \pm 5.2$ & $43.3 \pm 5.4$ & $33.4 \pm 3.9$ & $34.0 \pm 4.7$ & $68.9 \pm 3.5$ \\
\hline NP3061/UAS-shits1 & $49.6 \pm 5.8$ & $53.4 \pm 9.1$ & $37.7 \pm 4.3$ & $48.9 \pm 7.1$ & $35.1 \pm 6.8$ & $40.0 \pm 6.2$ & $72.3 \pm 3.0$ \\
\hline NP6024/+ & $48.2 \pm 3.7$ & $58.6 \pm 6.1$ & $45.2 \pm 4.3$ & $49.2 \pm 6.2$ & $39.2 \pm 3.1$ & $38.4 \pm 4.9$ & $71.6 \pm 4.0$ \\
\hline NP6024/+;; UAS-shis ${ }^{t s 1} /+$ & $53.5 \pm 3.2$ & $55.4 \pm 5.9$ & $41.7 \pm 6.2$ & $47.6 \pm 7.4$ & $39.5 \pm 7.2$ & $43.6 \pm 6.3$ & $74.1 \pm 4.3$ \\
\hline
\end{tabular}

No statistical difference was detected between the relevant groups $(n=8$, ANOVA). Average data are presented as means \pm SEM. 
A

Aversive olfactory conditioning

Non-HS group

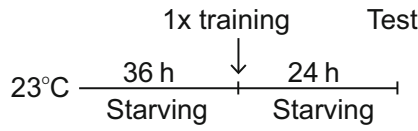

HS group:

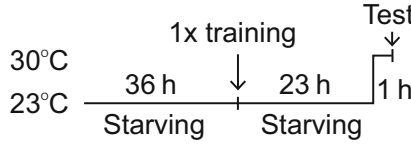

C $\square$ UAS-shits1/+

$\alpha / \beta$ surface and posterior (NP5286)
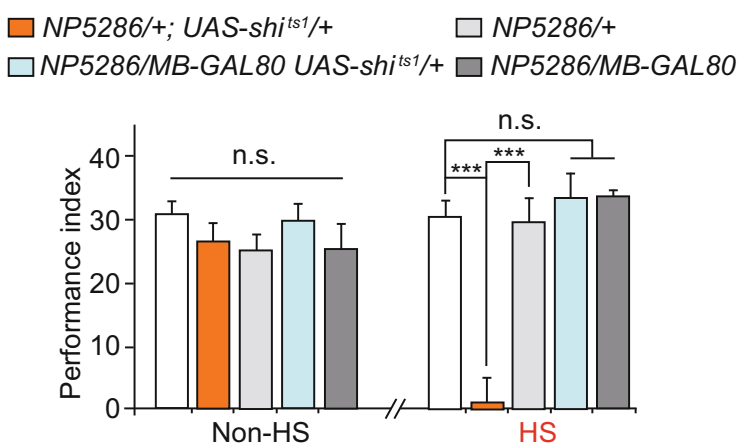
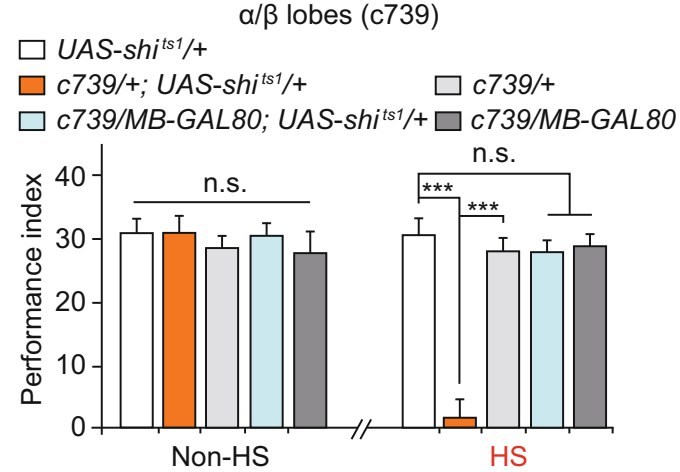

D $\alpha / \beta$ surface and posterior (NP3061)

$\square$ UAS-shits1/+

$\square N P 3061 /$ UAS-shits1 $\square N P 3061 /+$

MB-GAL80/+; NP3061/UAS-shits1 $\square$ MB-GAL80/+; NP3061/+ n.s.

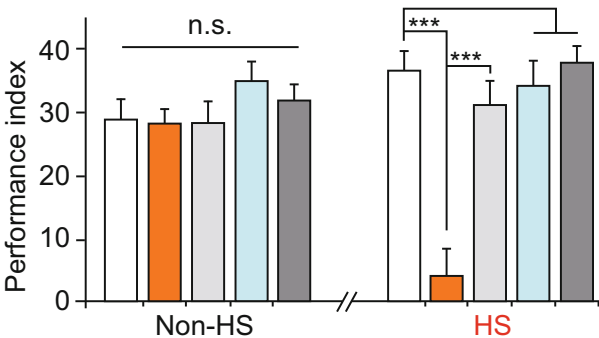

$\mathrm{E}$

$\alpha / \beta$ posterior (NP3208) and core (NP6024, NP7175)

$\square+/+; ;$ UAS-shit ${ }^{t s} /+$

$\square$ NP3208/+;; UAS-shits1/+ $\square$ NP6024/+;; UAS-shits1/+ $\square$ NP7175/+;; UAS-shits1/+

$\square$ NP3208/+ $\square$ NP6024/+ $\square$ NP7175/+

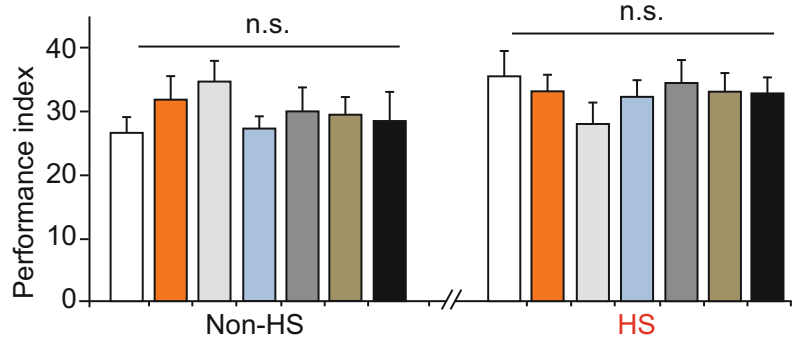

Figure 3. The synaptic output from a combination of MB $\alpha / \beta$ surface and posterior subdivisions is also necessary for retrieving appetitive LTM. (A) Temperature-shift protocols for non-HS and HS groups. Flies were food-deprived for $36 \mathrm{~h}$ before single-session appetitive training and for another $24 \mathrm{~h}$ after training. For non-HS group, flies were starved, trained and tested at $23^{\circ} \mathrm{C}$. For $\mathrm{HS}$ group, temperature was shifted from $23^{\circ} \mathrm{C}$ to $30^{\circ} \mathrm{C}$ at $1 \mathrm{~h}$ before testing.(B) $c 739$ labeled $M B \alpha / \beta$ region is necessary for appetitive LTM retrieval. In HS group, blocking synaptic output from $c 739$ labeled neurons disrupted LTM retrieval $(P<0.001, n=9-10$, ANOVA), while blocking non-MB $c 739$ neurons (by co-expressing MB-Gal80 and UAS-shis $\left.{ }^{t 51}\right)$ had no effect $(P>0.99, n=8-9$, ANOVA). In non-HS group, all genotypes showed normal LTM performance ( $P>0.53, n=8-10$, ANOVA and Kruskal-Walis ANOVA). (C) NP5286 labeled neurons (MB $\alpha / \beta$ surface and posterior) are required for appetitive LTM retrieval. In HS group, blocking synaptic output from NP5286 labeled neurons disrupted appetitive LTM retrieval $(P<0.001$, $n=9-10$, Kruskal-Walis ANOVA), while blocking non-MB NP5286 neurons (by co-expressing MB-Gal80 and UAS-shit $i^{t 1}$ ) had no effect $(P>$ $0.99, n=8-9$, ANOVA). In non-HS group, all genotypes showed normal LTM performance $(P>0.46, n=8-10$, ANOVA). (D) NP3061 labeled neurons (MB $\alpha / \beta$ surface and posterior) are required for appetitive LTM retrieval. In HS group, blocking synaptic output from NP3061 labeled neurons disrupted appetitive LTM retrieval $(P<0.001, n=9-10$, ANOVA), while blocking non-MB NP3061 neurons (by co-expressing MBGal80 and UAS-shis ${ }^{i s 1}$ ) had no effect $(P>0.99, n=8-9$, ANOVA). In non-HS group, all genotypes showed normal LTM performance $(P>0.22$ $n=8-10$, Kruskal-Walis ANOVA). (E) Output from either MB $\alpha / \beta$ posterior or core neurons are dispensable for appetitive LTM retrieval. In HS group, blocking synaptic output from MB $\alpha / \beta$ posterior subdivision (labeled by NP3208) or core subdivision (labeled by NP6024 and NP7175) alone had no effect on appetitive LTM retrieval $(P>0.50, n=8-10$, ANOVA). In non-HS group, all genotypes showed normal LTM performance $(P>0.36, n=8-10$, ANOVA). For the X chromosome-located NP3208, NP6024 and NP7175, only female results are shown. Average data are presented as means \pm SEM, ${ }^{* * *} P<0.001$, n.s. for non-significance. 

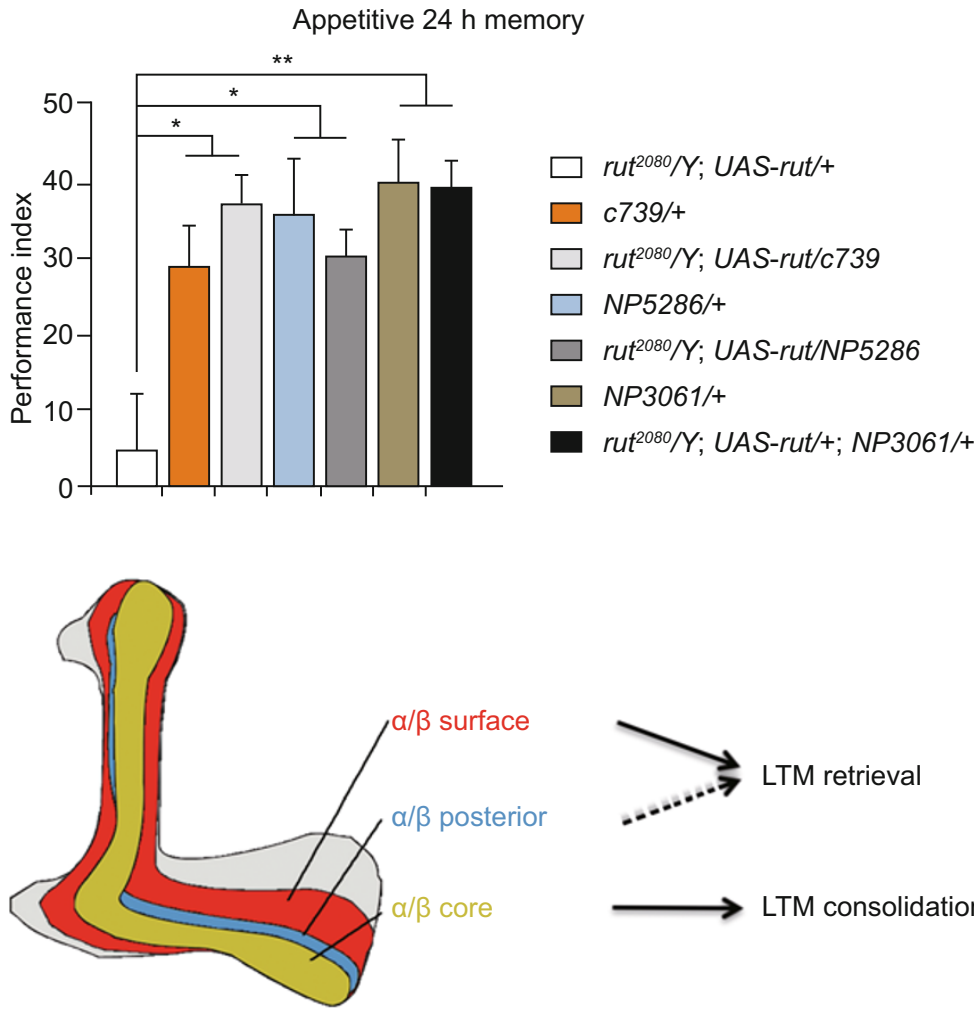

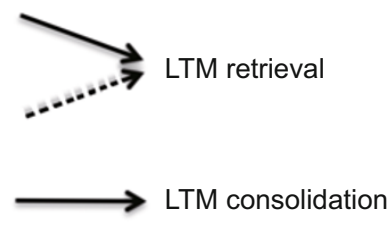

Figure 4. Restoring rut in $M B \alpha / \beta$ subdivisions is sufficient for appetitive LTM formation.c739driven rut expression fully rescues rut ${ }^{2080}$ LTM defect $(P<0.05, n=6-10$, ANOVA); NP5286-driven rut expression fully rescues rut ${ }^{2080}$ LTM defect $(P<0.05$, $n=6-10$, ANOVA); NP3061-driven rut expression fully rescues rut ${ }^{2080}$ LTM defect $(P<0.01, n=6-10$, ANOVA). Average data are presented as means \pm SEM, ${ }^{*} P<0.05,{ }^{* *} P<0.001$
Figure 5. Schematic of differential function of MB $\alpha / \beta$ subdivisions in LTM regulation. A combination of $M B \alpha / \beta$ surface and posterior neurons supports the LTM retrieval, whereas MB $\alpha / \beta$ core neurons permit LTM consolidation. used have the additional non-MB expression, we also used the $M B-G a / 80$ to rigorously examine the requirement for MB activity in these GAL4 lines. Our results confirmed the requirement of $c 739$ labeled $\alpha / \beta$ neurons in both aversive and appetitive LTM as reported previously (Isabel et al., 2004; Krashes et al., 2008; Trannoy et al., 2011) and further demonstrated output from a combination of $\alpha / \beta$ surface and posterior neurons is required for retrieving LTM, whereas the output from $\alpha / \beta$ posterior neurons or core neurons alone is clearly dispensable. Moreover, rut rescue data indicate the sufficiency of this combination of $M B \alpha / \beta$ neurons in rut-dependent LTM formation. Due to the lack of specific GAL4 line for targeting $\alpha / \beta$ surface neurons, we cannot specifically test the requirement of $\alpha / \beta$ surface region in LTM. Despite this caveat, it is still likely that MB $\alpha / \beta$ surface neurons serve a more important role than posterior neurons in LTM retrieval because of two lines of evidence: both NP5286 and NP3061 only have a weak expression in posterior subdivision (Tanaka et al., 2008), and the specific blockage of posterior neurons labeled by NP3208 had no effect on LTM.

We believe our data, taken with the previous report (Huang et al., 2012), have two significant implications. First, successfully retrieving both aversive and appetitive stable memory requires the support from a subgroup of about $500 \mathrm{MB} \alpha / \beta$ neurons; Second, a further functional partitioning exists within different anatomical subdivisions of $M B \alpha / \beta$ neuron in LTM processing: $M B \alpha / \beta$ core neurons permit LTM consolidation, whereas a combination of $\mathrm{MB} \alpha / \beta$ surface and posterior neurons supports the LTM retrieval (Fig. 5). These two implications could potentially shift our focus to a more restricted region for localizing the LTM engram in MB and add new circuitry components to the information processing loop underlying system consolidation of LTM (Dubnau and Chiang, 2013).

An immediate question raised here is, how do the different $\alpha / \beta$ neuron ensembles coordinate with each other to support $\mathrm{LTM}$ regulation. One possibility is one $\mathrm{MB} \alpha / \beta$ subdivision can interact with others during LTM processing. Although, to our knowledge, no evidence indicates different $M B$ neurons can form any synaptic connection at $\mathrm{MB}$ lobe region, one recent study does show the output from Kenyon cells transmits back to other compartments of MB through the presynaptic sites in calyx (Christiansen et al., 2011). Also, MB $\alpha / \beta$ subdivisions might communicate with each other through feedback loops constructed by various MB extrinsic neurons, such as DPM neurons and APL neurons (Lee et al., 1999; Keene et al., 2006; Wu et al., 2011; Pitman et al., 2011). Another possibility is that $M B \alpha / \beta$ subdivisions, being parallel with each other, release neurotransmitters to their common downstream neurons suggested for LTM storage, such as the DAL neurons (Chen et al., 2012) MB-V2 neurons (Séjourné et al., 2011), MB-V3 neurons (Pai et al., 2013) and the ellipsoid body (Wu et al., 2007), to regulate formation, consolidation and retrieval of LTM separately.

\section{MATERIALS AND METHODS}

Fly stocks and maintenance

GAL4 enhancer trap lines NP3061, NP5286, NP3208, NP6024 and NP7175 were kindly provided by Dr. Kei Ito. MB247-dsRed was ob- 
tained from Dr. André Fiala. MB-Gal80 was a gift from Dr. Scott Waddell. c739, UAS-mCD8::GFP, UAS-shits1 and rut ${ }^{2080} ;$ UAS-rut were obtained from Bloomington Stock Center (Bloomington, IN). To minimize the potential "leaky" effects of UAS-shis ${ }^{t s 1}$ expression during developmental stage, all flies were rared at $18^{\circ} \mathrm{C}$ with $60 \%$ humidity in a $12 \mathrm{~h}$ light-dark cycle on standard medium. All strains were outcrossed with $w^{1118}$ (isoCJ1) wild-type flies for five generations to minimize differences in genetic background.

\section{Confocal imaging}

3-day-old adult flies were anesthetized with $\mathrm{CO} 2$ and dissected in phosphate-buffered saline (PBS). The dissected brains were fixed in $4 \%$ paraformaldehyde in PBS for 30 min at room temperature followed by being washed in PBS for three times and then mounted in FocusClear (Pacgen Biopharmaceuticals). All images were acquired form a Zeiss 710 laser-scanning confocal microscope. Image stacks were obtained with Imaris 6.0 software (Bitplane, Zurich, Switzerland).

\section{Pavlovian olfactory aversive conditioning}

To generate aversive LTM, we used Pavlovian olfactory conditioning reinforced by electric shock. The training and testing procedures was performed in a room with $70 \%$ relative humidity and desired temperature as described previously (Tully et al., 1985, 1994). Two aversive odors, 3-octanol (OCT) (Fluka, $1.5 \times 10^{-3}$ dilution in heavy mineral oil), 4-methylcyclohexanol (MCH) (Fluka, $1 \times 10^{-3}$ dilution in heavy mineral oil) were used in all experiments.

For one training session, about 100 flies were exposed sequentially to the two odors (OCT and $\mathrm{MCH}$ ) for $60 \mathrm{~s}$ with a flush of fresh air for $45 \mathrm{~s}$ after each odor. Meanwhile, electric foot shock (twelve $1.5 \mathrm{~s}$ pulses with $3.5 \mathrm{~s}$ intervals; $60 \mathrm{~V}$ ) was delivered to flies during their exposure to the first odor (CS+), but not during exposure to the second odor (CS-). This training procedure was repeated for ten times with 15 min intervals between each session to generate a normal protein synthesis-dependent aversive LTM. After that, trained flies were transferred to food vials for a $24-\mathrm{h}$ resting at $18^{\circ} \mathrm{C}$ before testing.

To test memory, trained flies were transferred into the T-maze and allowed to choose between CS+ and CS- for $120 \mathrm{~s}$ at either $23^{\circ} \mathrm{C}$ or $30^{\circ} \mathrm{C}$ accordingly. The performance index $(\mathrm{PI})$ was calculated from the distribution of flies in the two T-maze arms (Tully et al., 1994). A $\mathrm{PI}$ of 0 indicates a distribution of 50:50 (no memory), while a PI of 100 indicates all the flies made a right choice (avoided the negatively reinforced odor). To eliminate any potential effect on the PI by odor bias, the experiments had a balanced design: each experiment $(n=1)$ includes two reciprocal groups with one used OCT as CS+ and the other one used $\mathrm{MCH}$ as $\mathrm{CS}+$. The final PI was the average of Pls from the two groups.

\section{Pavlovian olfactory appetitive conditioning}

The olfactory sugar rewarding appetitive-conditioning paradigm was performed as described previously (Krashes et al., 2008). The training tube (CS+) contained filter paper with dried sucrose that covered the entire wall of the training tube. The filter paper was made by applying a saturated sucrose solution and allowing it to dry before use. Another tube (CS-) was prepared with a dry filter paper. Before training, flies were food deprived for $36 \mathrm{~h}$ in vials containing a $2 \times 2 \mathrm{~cm}$ Watmann
$3 \mathrm{MM}$ filter paper soaked with $0.5 \mathrm{~mL}$ distilled water. During training, flies were transferred to the CS- tube and exposed to the CS- odor for $60 \mathrm{~s}$ followed by a-60 $\mathrm{s}$ flush of fresh air. Next, flies were moved to the CS+ tube and exposed to the CS+ odor for another $60 \mathrm{~s}$. After conditioning, flies were food-deprived again for another $24 \mathrm{~h}$. LTM was measured by the same method used for aversive memory testing.

\section{Sensori-motor responses}

To rule out the effect on sensori-motor abilities by any genetic manipulation, we also tested fly's olfactory acuity, shock reactivity and sucrose acuity as previously described (Tully et al., 1994; Kim et al., 2007). For olfactory acuity testing, odor avoidance responses were calculated by exposing about 100 naïve flies to the test odor (either OCT or $\mathrm{MCH}$, $1.5 \times 10^{-3}$ and $1 \times 10^{-3}$ dilution, respectively) versus fresh air in the T-maze. For shock reactivity testing, groups of about 100 untrained flies were exposed to two T-maze arms with $60 \mathrm{~V}$ electric foot shock delivered to one of the arms but not to the other. For sucrose acuity, about 100 untrained flies were subjected to two T-maze arms with a filter paper with dried sucrose in one of the arms and a dry filter paper in another arm. In all the three kinds of sensori-motor testing, flies were allowed to make choices between the two arms for $120 \mathrm{~s}$, after which they were trapped, anesthetized, and counted. A performance index (PI) was then quantified from the distribution of flies in two arms as that described in the memory test.

\section{Statistical analysis}

We used Shapiro-Wilk for the normality testing. Normally distributed data were subjected to one-way ANOVA followed by Bonferroni test (Origin version 8; OriginLab Corporation), while the non-normally distributed data were analyzed by Kruskal-Walis ANOVA. Statistical results are presented as means \pm SEM. Asterisks indicate critical values (*: $\left.P<0.05,{ }^{* *}: P<0.01,{ }^{* * *}: P<0.001\right)$. n.s. indicates no significant difference $(P>0.05)$.

\section{ACKNOWLEDGEMENTS}

We thank Dr. Kei Ito (University of Tokyo), Dr. André Fiala (Georg-August-University of Göttingen), Dr. Scott Waddell (University of Oxford) and the Bloomington Stock Center for providing fly strains. We are grateful for helpful discussions from other members in Yi Zhong lab. This work was supported by the National Basic Research Program (973 Program) (Nos. 2006CB500806 and 2009CB941301) and the Tsinghua University Initiative Scientific Research Program (all to Y.Z.). The authors declare no competing financial interests.

\section{COMPLIANCE WITH ETHICS GUIDELINES}

Cheng Huang, Pengzhi Wang, Zhiyong Xie, Lianzhang Wang, and Yi Zhong declare that they have no conflict of interest.

All institutional and national guidelines for the care and use of laboratory animals were followed.

\section{REFERENCES}

Aso, Y., Grubel, K., Busch, S., Friedrich, A.B., Siwanowicz, I., and Tanimoto, H. (2009). The mushroom body of adult Drosophila characterized by GAL4 drivers. J Neurogenet 23, 156-172. 
Blum, A.L., Li, W., Cressy, M., and Dubnau, J. (2009). Short- and longterm memory in Drosophila require cAMP signaling in distinct neuron types. Curr Biol 19, 1-10.

Chen, C.C., Wu, J.K., Lin, H.W., Pai, T.P., Fu, T.F., Wu, C.L., Tully, T., and Chiang, A.S. (2012). Visualizing long-term memory formation in two neurons of the Drosophila brain. Science 335, 678-685.

Christiansen, F., Zube, C., Andlauer, T.F., Wichmann, C., Fouquet, W., Owald, D., Mertel, S., Leiss, F., Tavosanis, G., Luna, A.F., et al. (2011). Presynapses in Kenyon cell dendrites in the mushroom body calyx of Drosophila. J Neurosci 31, 9696-9707.

Crittenden, J.R., Skoulakis, E.M., Han, K.A., Kalderon, D., and Davis, R.L. (1998). Tripartite mushroom body architecture revealed by antigenic markers. Learn Mem 5, 38-51.

de Belle, J.S., and Heisenberg, M. (1994). Associative odor learning in Drosophila abolished by chemical ablation of mushroom bodies. Science 263, 692-695.

Dubnau, J. and Chiang, A.S. (2013). System memory consolidation in Drosophila. Curr Opin Neurobiol 23, 84-91.

Dubnau, J., Grady, L., Kitamoto, T., and Tully, T. (2001). Disruption of neurotransmission in Drosophila mushroom body blocks retrieval but not acquisition of memory. Nature 411, 476-480.

Heisenberg, M. (2003). Mushroom body memoir: from maps to models. Nat Rev Neurosci 4, 266-275.

Huang, C., Zheng, X., Zhao, H., Li, M., Wang, P., Xie, Z., Wang, L., and Zhong, Y. (2012). A permissive role of mushroom body $\alpha / \beta$ core neurons in long-term memory consolidation in Drosophila. Curr Biol 22, 1981-1989.

Isabel, G., Pascual, A., and Preat, T. (2004). Exclusive consolidated memory phases in Drosophila. Science 304, 1024-1027.

Johard, H.A., Enell, L.E., Gustafsson, E., Trifilieff, P., Veenstra, J.A. and Nässel, D.R. (2008). Intrinsic neurons of Drosophila mushroom bodies express short neuropeptide $\mathrm{F}$ : relations to extrinsic neurons expressing different neurotransmitters. J Comp Neurol 507, 14791496.

Keene, A.C., Krashes, M.J., Leung, B., Bernard, J.A. and Waddell, S. (2006). Drosophila dorsal paired medial neurons provide a general mechanism for memory consolidation. Curr Biol 16, 1524-1530.

Kim, Y., Lee, H., and Han, K. (2007). D1 Dopamine Receptor dDA1 is required in the mushroom body neurons for aversive and appetitive learning in Drosophila, J Neurosci 27, 7640-7647.

Kitamoto, T. (2001). Conditional modification of behavior in Drosophila by targeted expression of a temperature-sensitive shibire allele in defined neurons. J Neurobiol 47, 81-92.

Krashes, M.J., DasGupta, S, Vreede, A., White, B., Armstrong, J.D. and Waddell, S. (2009). A neural circuit mechanism integrating motivational state with memory expression in Drosophila. Cell 139, 416-427.

Krashes, M.J., Keene, A.C., Leung, B., Armstrong, J.D., and Waddell, S. (2007). Sequential use of mushroom body neuron subsets during Drosophila odor memory processing. Neuron 53, 103-115.

Krashes, M.J., and Waddell, S. (2008). Rapid consolidation to a radish and protein synthesis-dependent long-term memory after singlesession appetitive olfactory conditioning in Drosophila. J Neurosci 28, 3103-3113.

Lee, T., Lee, A., and Luo, L. (1999). Development of the Drosophila mushroom bodies: sequential generation of three distinct types of neurons from a neuroblast. Development 126, 4065-4076.

Pai, T.P., Chen, C.C., Lin H.H., Chin A.L., Lai, J.S.Y., Lee, P.T., Tully, T. \& Chiang A.S. (2013). Drosophila ORB protein in two mushroom body output neurons is necessary for long-term memory formation. Proc Natl Acad Sci U S A 110, 7898-7903.

Perrat, P.N., DasGupta, S., Wang, J., Theurkauf, W., Weng, Z., Rosbash, M. and Waddell, S. (2013). Transposition-Driven genomic heterogeneity in the Drosophila brain. Science 340, 91-95.

Pitman, J.L., Huetteroth, W., Burke, C.J., Krashes, M.J., Lai, S.L., Lee, T. and Waddell, S. (2011). A pair of inhibitory neurons are required to sustain labile memory in the Drosophila mushroom body. Curr Biol 21, 855-861.

Qin, H., Cressy, M., Li, W., Coravos, J.S., Izzi, S.A., and Dubnau, J. (2012). Gamma neurons mediate dopaminergic input during aversive olfactory memory formation in Drosophila. Curr Biol 22, 608-614.

Séjourné, J., Plaçais, P.Y., Aso, Y., Siwanowicz, I., Trannoy, S, Thoma, V., Tedjakumala, S.R., Rubin, G.M. Tchénio, P., Ito, K., et al. (2011). Mushroom body efferent neurons responsible for aversive olfactory memory retrieval in Drosophila. Nat Neurosci 14, 903-910.

Silva, A.J., Zhou, Y., Rogerson, T., Shobe, J. and Balaji, J. (2009). Molecular and cellular approaches to memory allocation in neural circuits. Science 326, 391-395.

Sinakevitch, I., Grau, Y., Strausfeld, N.J. and Birman, S. (2010). Dynamics of glutamatergic signaling in the mushroom body of young adult Drosophila. Neural Dev 5, 10-30.

Small, S.A., Schobel, S.A., Buxton, R.B., Witter, M.P. and Barnes, C.A. (2011). A pathophysiological framework of hippocampal dysfunction in ageing and disease. Nat Rev Neurosci 12, 585-601.

Tanaka, N.K., Tanimoto, H., and Ito, K. (2008). Neuronal assemblies of the Drosophila mushroom body. J Comp Neurol 508, 711-755.

Trannoy, S., Redt-Clouet, C., Dura, J.M., and Preat, T. (2011). Parallel processing of appetitive short- and long-term memories in Drosophila. Curr Biol 21, 1647-1653.

Tully, T., Preat, T., Boynton, S.C., and Del Vecchio, M. (1994). Genetic dissection of consolidated memory in Drosophila. Cell 79, 35-47.

Tully, T., and Quinn, W.G. (1985). Classical conditioning and retention in normal and mutant Drosophila melanogaster. J Comp Physiol [A] 157, 263-277.

Tye, K.M. and Deisseroth, K. (2012). Optogenetic investigation of neural circuits underlying brain disease in animal models. Nat Rev Neurosci 13, 251-266.

van Strien, N.M., Cappaert, N.L. and Witter, M.P. (2009). The anatomy of memory: an interactive overview of the parahippocampal-hippocampal network. Nat Rev Neurosci 10, 272-282.

Wang, Y., Mamiya, A., Chiang, A., and Zhong, Y. (2008). Imaging of an Early Memory Trace in the Drosophila Mushroom Body. J Neurosci 28, 4368-4376.

Wu, C.L., Shih, M.F., Lai, J.S., Yang, H.T., Turner, G.C., Chen, L. and Chiang, A.S. (2011). Heterotypic gap junctions between two neurons in the drosophila brain are critical for memory. Curr Biol 21, 848-854.

Wu, C.L., Xia, S., Fu, T.F., Wang, H., Chen, Y.H., Leong, D., Chiang, A.S. and Tully, T. (2007). Specific requirement of NMDA receptors for long-term memory consolidation in Drosophila ellipsoid body. Nat Neurosci 10, 1578-1586. 